\title{
Impedance scaling for small angle transitions
}

\author{
G. Stupakov and K. L.F. Bane \\ SLAC National Accelerator Laboratory, Stanford University, Stanford, California 94309, USA
}

\section{Zagorodnov}

Deutsches Elektronen-Synchrotron, Notkestrasse 85, 22603 Hamburg, Germany

(Received 24 September 2010; published 10 January 2011)

\begin{abstract}
Based on the parabolic equation approach to Maxwell's equations, we have derived scaling properties of impedance that apply either to (1) structures of general shape at high frequencies, or (2) small angle transitions at all frequencies. Applying these scaling properties to impedance/wakefield calculation of long, small angle, beam pipe transitions, like one often finds in insertion regions of storage rings, one can greatly reduce the computer resource required. We have tested the scaling with wakefield simulations of 2D and 3D models of such transitions, and have found that it appears to work well.
\end{abstract}

DOI: 10.1103/PhysRevSTAB.14.014402

PACS numbers: 41.60.- m, 03.50.De

\section{INTRODUCTION}

In modern ring-based light sources, one often finds insertion devices having extremely small vertical apertures (on the order of millimeters) to allow for maximal undulator fields reaching the beam. Such insertion devices require that there be beam pipe transitions from these small apertures to the larger cross sections (normally on the order of centimeters) found in the rest of the ring. The fact that there may be many such transitions, and that these transitions introduce beam pipe discontinuities very close to the beam path, means that their impedance will be large and, in fact, may dominate the impedance budget of the entire ring. To reduce their impact on impedance, the transitions are normally tapered gradually over a long distance. The accurate calculation of the impedance or wakefield of these long transitions, which are typically 3D objects (i.e. they do not have cylindrical symmetry), can be quite a challenging numerical task.

In this paper we present a method of obtaining the impedance of a long, small angle transition from the calculation of a scaled, shorter one. Normally, the actual calculation is obtained from a time domain simulation of the wakefield in the structure, where the impedance can be obtained by performing a Fourier transform. We shall see that the scaled calculation reduces the computer time and memory requirements significantly, especially for 3D problems, and can make the difference between being able to solve a problem or not. The method is based on the parabolic equation approach to solving Maxwell's equation developed in Refs. [1,2].

This report is organized as follows: We begin by developing the theory of impedance scaling for small angle transitions. This is followed by numerical calculations to test the validity of the theory. Our examples consist of longitudinally symmetric transitions that connect a large beam pipe to a small one and then back again by means of small angle tapers. They include (i) a 2D (cylindrically symmetric) transition with a short central section (similar to a collimator), (ii) 2D transitions with longer central pipes, and (iii) a 3D transition with long central pipe. We finish with a concluding section. To differentiate the three types of examples we call them short (i), long (ii), and $3 D$ (iii) transitions.

\section{THEORY}

We start from the parabolic equation formulated in [3]. As discussed in [1], in the general case of arbitrary angles this equation is valid for frequencies $\omega \gg c / a$, where $a$ is a characteristic transverse size of the pipe. However, for small angle tapers and collimators, the region of validity of this equation extends toward smaller frequencies and includes $\omega \leqslant c / a$.

The parabolic equation is formulated for the envelope part of the electromagnetic field,

$$
\hat{\boldsymbol{E}}(x, y, z, \omega)=\int_{-\infty}^{\infty} d t \mathrm{e}^{i \omega t-i k z} \boldsymbol{E}(x, y, z, t),
$$

where $k=\omega / c$. It is written in terms of the transverse component $\hat{\boldsymbol{E}}_{\perp}=\left(\hat{E}_{x}, \hat{E}_{y}\right)$ of the vector $\hat{\boldsymbol{E}}$,

$$
k \frac{\partial}{\partial z} \hat{\boldsymbol{E}}_{\perp}=\frac{i}{2}\left(\nabla_{\perp}^{2} \hat{\boldsymbol{E}}_{\perp}-\frac{4 \pi}{c} \nabla_{\perp} \hat{j}_{z}\right),
$$

where $z$ is the coordinate in the direction of motion of the beam, and $\hat{j}_{z}$ is the Fourier transformed projection of the beam current along $z$ :

$$
\hat{j}_{z}(x, y, z, \omega)=\int_{-\infty}^{\infty} d t \mathrm{e}^{i \omega t-i k z} j_{z}(x, y, z, t) .
$$

The longitudinal electric field $\hat{E}_{z}$ is expressed in terms of $\hat{E}_{\perp}$ :

$$
\hat{E}_{z}=\frac{i}{k}\left(\nabla_{\perp} \cdot \hat{\boldsymbol{E}}_{\perp}-\frac{4 \pi}{c} \hat{j}_{z}\right)
$$


We assume perfect conductivity of the metal walls. The boundary condition for the electric field requires a vanishing tangential component on the wall,

$$
\boldsymbol{n} \times\left.\hat{\boldsymbol{E}}\right|_{w}=0,
$$

where $\boldsymbol{n}$ is the normal vector to the surface of the wall.

The current $\hat{j}_{z}$ in Eqs. (2) and (4) corresponds to a unit point charge moving with the speed of light along the axis of the system $x=y=0, j_{z}=q c \delta(x) \delta(y) \delta(z-c t)$. It is given by the following expression:

$$
\hat{j}_{z}=q \delta(x) \delta(y) \text {. }
$$

The longitudinal impedance on the $z$ axis at frequency $\omega$ is given by

$$
Z_{\|}(\omega)=-\frac{1}{q} \int_{-\infty}^{\infty} d z \hat{E}_{z}(0,0, z, \omega) .
$$

Because of the presence of $\delta$ functions in the current (6), the electric field $\hat{\boldsymbol{E}}$ has a singularity on the axis $z$. An equivalent formulation of the impedance problem which avoids the singular terms is the following. We introduce the vacuum electric field $\hat{\boldsymbol{E}}_{\mathrm{vac}}$ of the current $\hat{j}_{z}$ and subtract it from $\hat{E}$ :

$$
\hat{\mathcal{E}}=\hat{\boldsymbol{E}}-\hat{\boldsymbol{E}}_{\mathrm{vac}} .
$$

The vacuum field is the beam field in the absence of material boundaries in the problem; it satisfies the same Eq. (2), but is not required to satisfy the boundary condition (5). While it is easy to write down an analytical expression for $\hat{\boldsymbol{E}}_{\mathrm{vac}}$, we will not need it in what follows. The equations for $\hat{\mathcal{E}}$, which we call the radiation field, are

$$
k \frac{\partial}{\partial z} \hat{\mathcal{E}}_{\perp}=\frac{i}{2} \nabla_{\perp}^{2} \hat{\mathcal{E}}_{\perp}, \quad \hat{\mathcal{E}}_{z}=\frac{i}{k} \nabla_{\perp} \cdot \hat{\mathcal{E}}_{\perp},
$$

with the boundary condition

$$
\boldsymbol{n} \times\left.\hat{\boldsymbol{E}}\right|_{w}+\boldsymbol{n} \times\left.\hat{\mathcal{E}}_{\mathrm{vac}}\right|_{w}=0 .
$$

The vacuum electric field is perpendicular to the direction of motion (because we consider an ultrarelativistic point charge), and does not contribute to the impedance. Note also that the vacuum field does not depend on $z, \hat{\boldsymbol{E}}_{\mathrm{vac}}(x, y)$.

Let us assume that the geometry of a given surface of the metallic wall is determined by the equation $U(x, y, z)=0$. Instead of considering one particular shape of the pipe, we consider a family of such pipes, which are defined by various scale lengths $\lambda$ in the longitudinal direction. This means that $U$ is also a function of the parameter $\lambda$, and it has a special dependence on $\lambda$ :

$$
U(x, y, z ; \lambda)=V\left(x, y, \frac{z}{\lambda}\right) .
$$

Increasing the parameter $\lambda$ in Eq. (11), we extend the pipe in the $z$ direction without changing its transverse shape at each cross section, while decreasing $\lambda$ contracts the pipe along $z$.

We now define the normal vector to the surface of the pipe, $\boldsymbol{n}=\nabla U$ or

$$
\boldsymbol{n}=\boldsymbol{e}_{x} \frac{\partial V}{\partial x}+\boldsymbol{e}_{y} \frac{\partial V}{\partial y}+\boldsymbol{e}_{z} \frac{1}{\lambda} \frac{\partial V}{\partial \zeta},
$$

where we have introduced the dimensionless scaled coordinate $\zeta=z / \lambda$, and used notations $\boldsymbol{e}_{x}, \boldsymbol{e}_{y}$, and $\boldsymbol{e}_{z}$ for unit vectors in respective directions. We will indicate the dependence of fields versus the parameter $\lambda$ by adding $\lambda$ to the list of arguments and separating it by the semicolon, e.g., $\hat{\mathcal{E}}_{\perp}(x, y, z, \omega ; \lambda)$.

Our goal now is to prove that a solution to the parabolic equation depends on the coordinate $z$ only through the dimensionless variable $\zeta$; more precisely, we will prove that

$$
\begin{aligned}
& \hat{\mathcal{E}}_{\perp}(x, y, z, \omega ; \lambda)=\boldsymbol{F}_{\perp}\left(x, y, \frac{z}{\lambda}, \frac{\omega}{\lambda}\right), \\
& \hat{\mathcal{E}}_{z}(x, y, z, \omega ; \lambda)=\frac{1}{\lambda} G\left(x, y, \frac{z}{\lambda}, \frac{\omega}{\lambda}\right),
\end{aligned}
$$

where $\boldsymbol{F}_{\perp}$ and $G$ are functions of four arguments. To prove this statement, we first need to show that substituting Eqs. (13) into our equations and the boundary condition results in expressions which involve the coordinate $z$, the parameter $\lambda$, and the wave number $k$ as combinations $z / \lambda$ and $k / \lambda$ only. Indeed, substituting (13) into Eqs. (9) we find

$$
\frac{k}{\lambda} \frac{\partial}{\partial \zeta} \boldsymbol{F}_{\perp}=\frac{i}{2} \nabla_{\perp}^{2} \boldsymbol{F}_{\perp}, \quad G=\frac{i \lambda}{k} \nabla_{\perp} \cdot \boldsymbol{F}_{\perp},
$$

which clearly satisfies our requirement.

We now take a close look at the boundary condition (10). Rewriting it in terms of the perpendicular and transverse components of the field, we obtain (remember that $\hat{\boldsymbol{E}}_{\mathrm{vac}}$ has only perpendicular components)

$$
\begin{aligned}
n_{z} \boldsymbol{e}_{z} \times & \left.\hat{\mathcal{E}}_{\perp}\right|_{w}+\boldsymbol{n}_{\perp} \times\left.\hat{\mathcal{E}}_{\perp}\right|_{w}+\boldsymbol{n}_{\perp} \times\left.\boldsymbol{e}_{z} \hat{\mathcal{E}}_{z}\right|_{w} \\
& +n_{z} \boldsymbol{e}_{z} \times\left.\hat{\boldsymbol{E}}_{\mathrm{vac}}\right|_{w}+\boldsymbol{n}_{\perp} \times\left.\hat{\boldsymbol{E}}_{\mathrm{vac}}\right|_{w}=0,
\end{aligned}
$$

where $\boldsymbol{n}_{\perp}=\boldsymbol{n}-\boldsymbol{e}_{z}\left(\boldsymbol{e}_{z} \cdot \boldsymbol{n}\right)$ is the perpendicular to $z$ part of the vector $\boldsymbol{n}$. The first, third, and fourth terms in this equation are perpendicular to $\boldsymbol{e}_{z}$, and the second and fifth terms are directed in the $z$ direction. Hence, they can be split into two separate equations. The first one is

$$
\begin{aligned}
& n_{z} \boldsymbol{e}_{z} \times\left.\hat{\mathcal{E}}_{\perp}\right|_{w}+\boldsymbol{n}_{\perp} \times\left.\boldsymbol{e}_{z} \hat{\mathcal{E}}_{z}\right|_{w}+n_{z} \boldsymbol{e}_{z} \times\left.\hat{\boldsymbol{E}}_{\mathrm{vac}}\right|_{w} \\
& =\frac{1}{\lambda} \frac{\partial V}{\partial \zeta} \boldsymbol{e}_{z} \times\left.\hat{\boldsymbol{F}}_{\perp}\right|_{w}+\frac{1}{\lambda} \boldsymbol{n}_{\perp} \times\left.\boldsymbol{e}_{z} G\right|_{w} \\
& \quad+\frac{1}{\lambda} \frac{\partial V}{\partial \zeta} \boldsymbol{e}_{z} \times\left.\hat{\boldsymbol{E}}_{\mathrm{vac}}\right|_{w}=0 .
\end{aligned}
$$

The last line, after cancellation of the factor $1 / \lambda$, clearly shows that the parameter $\lambda$ does not enter explicitly into it. 
The second boundary equation is

$$
\begin{gathered}
\boldsymbol{n}_{\perp} \times\left.\hat{\mathcal{E}}_{\perp}\right|_{w}+\boldsymbol{n}_{\perp} \times\left.\hat{\boldsymbol{E}}_{\mathrm{vac}}\right|_{w}=\boldsymbol{n}_{\perp} \times\left.\hat{\boldsymbol{F}}_{\perp}\right|_{w} \\
+\boldsymbol{n}_{\perp} \times\left.\hat{\boldsymbol{E}}_{\mathrm{vac}}\right|_{w}=0,
\end{gathered}
$$

and it again does not explicitly contain the parameter $\lambda$. Our statement is therefore proved.

Substituting the second of Eqs. (13) into (7), we find the scaling property for the longitudinal impedance,

$$
Z_{\|}(\omega ; \lambda)=R\left(\frac{\omega}{\lambda}\right)
$$

where $R$ is a function of one variable.

Taking the Fourier transform of the impedance we find, in addition, that the longitudinal wake scales as

$$
w(s ; \lambda)=\lambda u(s \lambda),
$$

where $u$ is a function of one variable.

For the transverse forces the results are similar. The high-frequency impedance scales as

$$
\mathbf{Z}_{\perp}(\omega ; \lambda)=\frac{1}{\lambda} \mathbf{R}_{\perp}\left(\frac{\omega}{\lambda}\right)
$$

where $\mathbf{R}_{\perp}$ is a function of one variable; and for the transverse wake is

$$
\mathbf{w}_{\perp}(s ; \lambda)=\mathbf{u}_{\perp}(s \lambda),
$$

where $\mathbf{u}_{\perp}$ is a function of one variable.

\section{COMPARISON WITH SOME IMPEDANCE MODELS}

As is pointed out at the beginning of the previous section, the parabolic equation, and hence our results (18)-(21) are valid in either a high-frequency limit, or, for small angle transitions, for arbitrary frequencies. It is instructive to test this statement by comparing it with analytical expressions for the impedance in some limiting cases available from the literature. Note that by small angle here we mean that the $z$ component of the normalized normal vector to the surface of the wall is small, $n_{z} /|\boldsymbol{n}| \ll 1$. In the case of axisymmetric transitions with the pipe radius $b(z)$ it reduces to $\left|b^{\prime}\right| \ll 1$, where $b^{\prime}$ denotes the derivative of $b$ with respect to $z$.

One such special case is a pair of small angle, axisymmetric transitions at low frequencies, for which the solution was found by Yokoya [4]. The applicability condition for this case was found in [5] and is given by $k b^{2}\left|b^{\prime}\right| \ll \Delta b$, where $\Delta b$ is the scale of variation of the radius through the transition. The longitudinal impedance in this limit is purely imaginary and is given by

$$
Z_{\|}(\omega)=-\frac{i \omega Z_{0}}{4 \pi c} \int_{-\infty}^{\infty} d z\left(b^{\prime}\right)^{2},
$$

which clearly satisfies the scaling (18).
Our second example refers to the longitudinal impedance of a deep pillbox cavity (connected to beam pipes) in the high-frequency limit. The model is called "the diffraction model" and $Z_{\|}$is given by $[6,7]$

$$
Z_{\|}(\omega)=\frac{Z_{0}}{2 \pi} \frac{(1+i)}{\sqrt{\pi} b} \sqrt{\frac{c L}{\omega}},
$$

where $L$ is the length of the cavity and $b$ is the radius of incoming and outgoing pipes. By "deep" we require that $k(d-b)^{2} \geq L$, where $d$ is the radius of the cavity. Again, it is easy to see that (23) satisfies the scaling (18). In this case, there is no requirement on smallness of the transition angle; however, there is one on frequency: Eq. (23) is valid in the limit of high frequencies, when $L \ll k b^{2}$.

Finally, we point out that the so-called "optical model" of impedance developed in [8,9], valid for arbitrary 3D transitions again in the limit of high frequencies, also satisfies (18). Indeed, in the optical model the longitudinal impedance is purely real and depends neither on frequency nor on the length of the transition (it only depends on the geometrical shape of the transition projected along the axis $z$ of the system), and hence is invariant with respect to the scaling of the coordinate $z$ and the frequency $\omega$ in (18). One 2D example of the optical model is the impedance of the shallow cavity [same geometry as the deep cavity, except that $\left.k(d-b)^{2} \lesssim L\right]$ at high frequency, where [10]

$$
Z_{\|}(\omega)=\frac{Z_{0}}{\pi} \ln \frac{d}{b} .
$$

For all the examples of this section, formulas exist also for the transverse impedance, and one can similarly show that they satisfy Eq. (20).

We would like to emphasize here that, while the scalings derived in Sec. II do not necessarily require structures with small angles (as was demonstrated by the previous two examples), the main focus of this paper is the case of small angle transitions, where using the scalings can significantly reduce the computer resources required in numerical calculations of the wakes. In the next section we give several examples of small angle transitions, and compare numerical calculations of impedance/wakes for the original structure with those for a scaled structure, in order to demonstrate the validity of the scaling presented in this report.

\section{SCALED CALCULATIONS}

To test the impedance scaling, we perform numerical simulation, using 2D and 3D versions of the time-domain, finite difference Maxwell equation solver ECHO [11]. The program finds wakefields excited by a speed-of-light Gaussian bunch of finite length $\sigma_{z}$ in a structure, such as a beam pipe transition. The impedance, in turn, is found by Fourier transforming the wake and dividing by the bunch spectrum. For the combination of a short bunch and a long, 
small angle transition, one can greatly reduce the requirements on computer time and memory by solving the scaled problem, i.e. the one with the longitudinal dimension scaled by a factor $\lambda<1$, and the bunch length scaled by $1 / \lambda$.

In simulations we compare the wakefield and impedance computed for two structures. The first one, which we call nominal, has a length $L$, and the second one, which we call scaled, has a length $\lambda L$, and is obtained from the first one by scaling along $z$, as described in the previous section. Typically, in this paper, we choose $\lambda=\frac{1}{2}$. We denote $W\left(s, \sigma_{z}\right)$ the wake of the nominal structure, and $W_{\lambda}\left(s, \sigma_{z}\right)$ the wake of the scaled one. Using (19) it is easy to establish a relation between these two functions by convolving the bunch distribution with the wake of a point charge $w(s ; \lambda)$. For the nominal structure we set $\lambda=1$ and obtain

$$
\begin{aligned}
W\left(s, \sigma_{z}\right) & =\frac{1}{\sqrt{2 \pi} \sigma_{z}} \int_{0}^{\infty} d s^{\prime} w\left(s^{\prime} ; 1\right) e^{-\left(s-s^{\prime}\right)^{2} / 2 \sigma_{z}^{2}} \\
& =\frac{1}{\sqrt{2 \pi} \sigma_{z}} \int_{0}^{\infty} d s^{\prime} u\left(s^{\prime}\right) e^{-\left(s-s^{\prime}\right)^{2} / 2 \sigma_{z}^{2}}
\end{aligned}
$$

for the scaled structure we have

$$
\begin{aligned}
W_{\lambda}\left(s, \sigma_{z}\right) & =\frac{\lambda}{\sqrt{2 \pi} \sigma_{z}} \int_{0}^{\infty} d s^{\prime} w\left(s^{\prime} ; \lambda\right) e^{-\left(s-s^{\prime}\right)^{2} / 2 \sigma_{z}^{2}} \\
& =\frac{\lambda}{\sqrt{2 \pi} \sigma_{z}} \int_{0}^{\infty} d s^{\prime} u\left(s^{\prime} \lambda\right) e^{-\left(s-s^{\prime}\right)^{2} / 2 \sigma_{z}^{2}}
\end{aligned}
$$

Changing the integration variable to $\zeta=s^{\prime} \lambda$ in the last integral we find that

$$
W\left(s, \sigma_{z}\right)=\frac{1}{\lambda} W_{\lambda}\left(\frac{s}{\lambda}, \frac{\sigma_{z}}{\lambda}\right)
$$

For the transverse wake we have the relation

$$
\mathbf{W}_{\perp}\left(s, \sigma_{z}\right)=\mathbf{W}_{\perp \lambda}\left(\frac{s}{\lambda}, \frac{\sigma_{z}}{\lambda}\right)
$$

The maximum mesh size and time step in the wake calculations are limited to a specific fraction of $\sigma_{z}$ and $\sigma_{z} / c$, respectively (the mesh size is typically taken to be $\sigma_{z} / 5$ ). Let us assume that the total range in $s$ over which the wake is needed is short compared to the total structure length (which for electron machines and small angle transitions typically is true). Let us first consider a 2D transition. Since the bunch length in the scaled calculation is increased by $1 / \lambda$, the calculation window (that moves with the beam) has a factor $\lambda$ fewer mesh points in the radial direction, but the same number of mesh points in the longitudinal one [the mesh density is lower but the window needs to be $1 / \lambda$ times longer-see Eq. (27)]. In addition, the structure length for the scaled calculation is reduced by

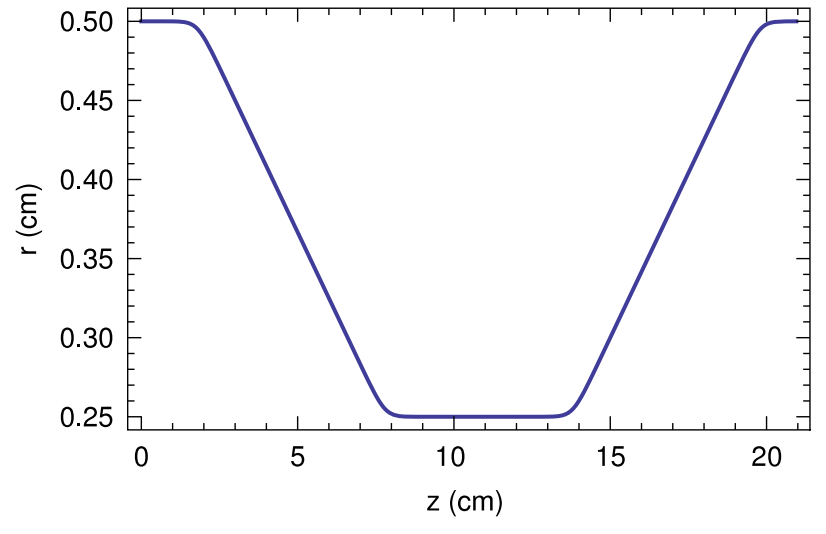

FIG. 1. Geometry for smooth, 2D example problem.

the factor $\lambda$; and since the mesh is coarser, the number of time steps for the beam to traverse the structure is reduced by $\sim \lambda^{2}$. Thus, we see that the scaled calculation reduces the computation time (in $2 \mathrm{D}$ problems) by $\lambda^{3}$; in a $3 \mathrm{D}$ example the factor becomes $\lambda^{4}$, a significant speed-up even if we scale only by a factor of $2\left(\lambda=\frac{1}{2}\right)$.

\section{NUMERICAL EXAMPLES}

\section{A. Short transition}

We begin with a 2D, smooth test example that has a central beam pipe that is relatively short; i.e., it looks more like a collimator than an insertion device transition (see Fig. 1). A beam pipe of radius $b=5 \mathrm{~mm}$ is connected by two gentle, symmetric transitions to a beam pipe of radius $a=2.5 \mathrm{~mm}$. Although the boundary is everywhere smooth, the tapers approximate straight lines of angle $\theta=2.4^{\circ}$, with a central pipe length of $6 \mathrm{~cm}$. For the nominal calculation the bunch length is $\sigma_{z}=0.1 \mathrm{~mm}$, and the mesh size is $0.01 \mathrm{~mm}$. For the scaled calculation, we take $\lambda=\frac{1}{2}$, the structure is half as long, and the bunch length and mesh size are twice as large.

The real and imaginary part of the impedance for the nominal and scaled structures are shown in Fig. 2, and the wakefield of the bunch in Fig. 3. One can see very good agreement between the nominal and the scaled structure results. Finally, we should also mention that when we replace the smooth collimator geometry with one consisting of straight line segments (in longitudinal view) the results remain essentially unchanged.

\section{B. Long transition}

In typical insertion devices, the central beam pipe has an aperture on the order of millimeters and a length on the order of meters. Our second example transition is a 2D model of such a transition: in longitudinal view there are straight line tapers of (small) angle $\theta$ that connect a large beam pipe of radius $b$ to a very long pipe of smaller radius $a$. We assume that the impedance of such a structure is given by the sum of the impedance of a step-in transition 

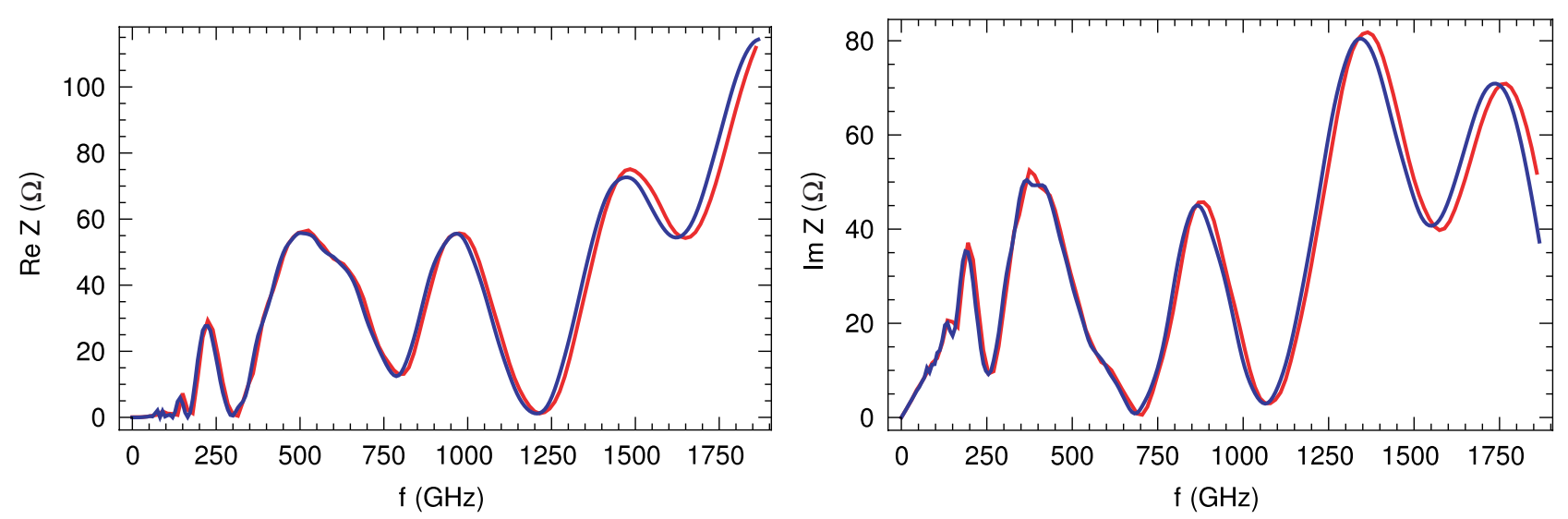

FIG. 2. Real (left) and imaginary (right) parts of impedance for nominal and scaled structures. The blue curves show the impedance of the nominal structure, and the red curves show the impedance of the structure that is half as long scaled using Eq. (18).

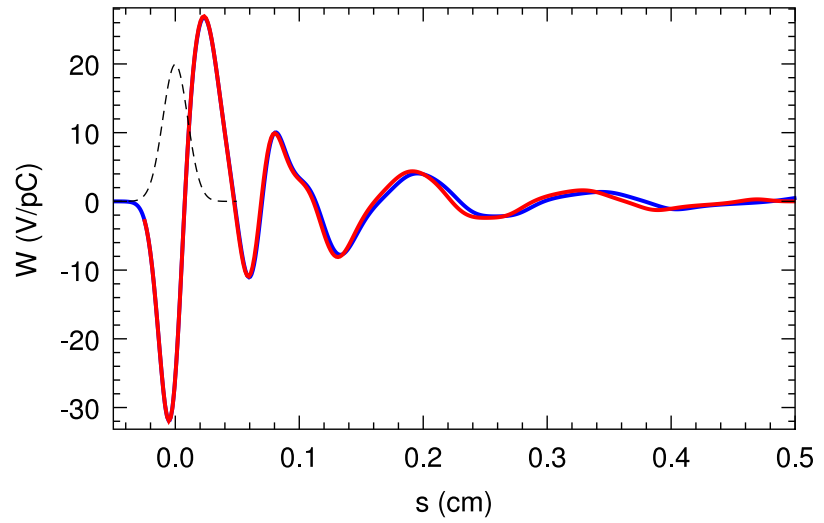

FIG. 3. Comparison of the wakefields of a Gaussian bunch for the nominal and scaled structures. The blue curve shows the wake excited by a bunch with $\sigma_{z}=0.1 \mathrm{~mm}$ in the nominal structure, and the red curve shows the wake in the structure that is half as long excited by the bunch with $\sigma_{z}=0.2 \mathrm{~mm}$ and scaled using Eq. (27).

(from $b$ to $a$, with $b>a$ ) plus the impedance of a step-out transition (from $a$ to $b$ ) (see, e.g., Ref. [10]). In the 2D case we can write

$$
\begin{gathered}
Z_{\text {in }}=Z_{1}-Z_{s}, \quad Z_{\text {out }}=Z_{1}+Z_{s}, \\
Z_{s}=\frac{Z_{0}}{2 \pi} \ln \left(\frac{b}{a}\right),
\end{gathered}
$$

where $Z_{s}$ is a potential term. For a longitudinally symmetric transition

$$
Z \approx Z_{\text {in }}+Z_{\text {out }}=2\left(Z_{\text {in }}+Z_{s}\right),
$$

and similarly the wake $W \approx 2\left(W_{\text {in }}+W_{s}\right)$, with $W_{s}=$ $-Z_{s} c \lambda_{z}$. Thus, we can obtain the impedance of the symmetric transition from the numerical calculation of just the step-in problem.

As specific example we take a $2 \mathrm{D}$ model of wiggler transitions that have been considered for PEP-X [12]. For

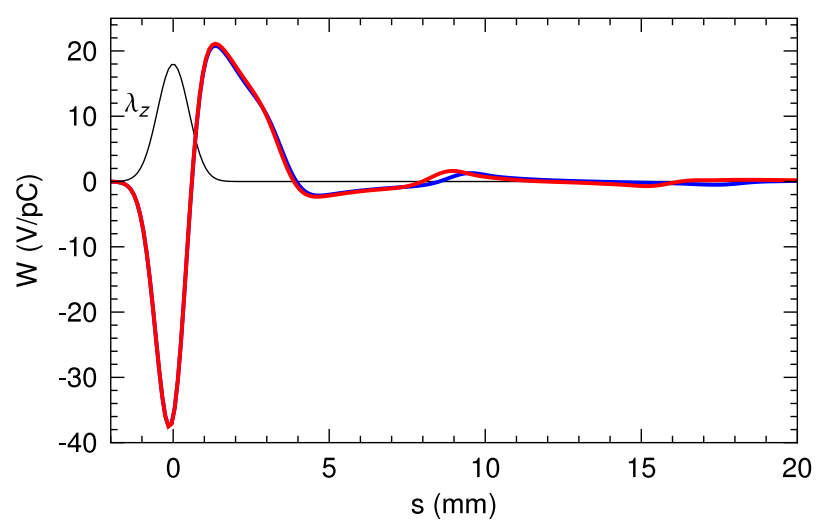

FIG. 4. Longitudinal wake for $\sigma_{z}=0.5 \mathrm{~mm}$ bunch in $2 \mathrm{D}$ model of PEP-X transitions (blue) and results obtained from the scaled problem with $\lambda=\frac{1}{2}$ (red). The nominal bunch shape is also given with the head to the left (black).

the purpose of generating a pseudo-Green function wake, the wake of all ring elements was needed for a Gaussian bunch with $\sigma_{z}=0.5 \mathrm{~mm}$ to a distance of $60 \mathrm{~mm}$ behind the bunch (an extremely challenging task for the 3D wiggler transitions). The 2D model that we consider is a symmetric transition with $a=7.5 \mathrm{~mm}, b=48 \mathrm{~mm}$, and $\theta=5.8^{\circ}$ (we take as nominal bunch length for the calculation $\sigma_{z}=0.5 \mathrm{~mm}$ ). In the real structure the central beam pipe is meters long; for our calculations we take it to be infinitely long. The wake and the scaled $\left(\lambda=\frac{1}{2}\right)$ results are given in Fig. 4. We see that the wake is simpler, with less oscillation than for the finite-length example above. We, in addition, see that the agreement between the wake and the scaled wake is quite good.

In the transverse case the wake scales as Eq. (28). For a symmetric transition with long bottom, the results can again be obtained from a step-in wake calculation alone. The longitudinal dipole wake $W_{z}^{(1)}=2\left(W_{\text {in }}^{(1)}+W_{s}^{(1)}\right)$, $W_{s}^{(1)}=-Z_{s}^{(1)} c \lambda_{z}$, with the static impedance given by 


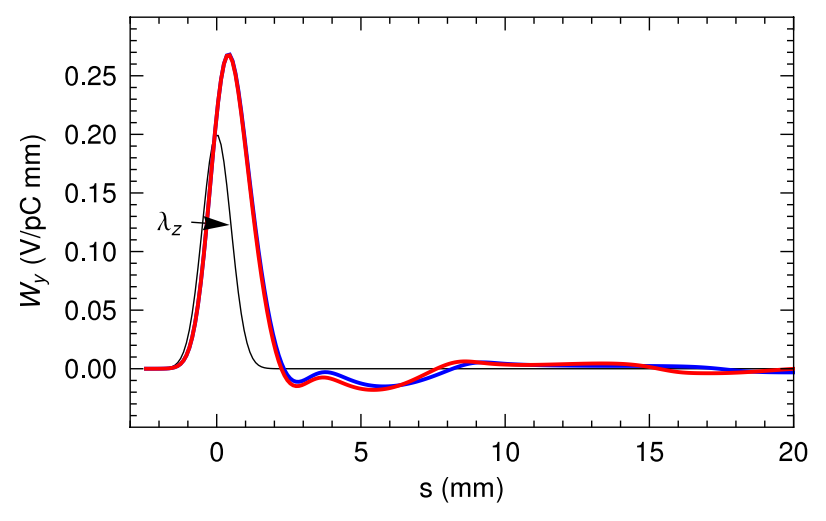

FIG. 5. Transverse wake for $\sigma_{z}=0.5 \mathrm{~mm}$ bunch in 2D model of PEP-X transitions (blue) and results obtained from the scaled problem with $\lambda=\frac{1}{2}$ (red). The nominal bunch shape is also given with the head to the left (black).

$$
Z_{s}^{(1)}=\frac{Z_{0}}{2 \pi}\left(\frac{1}{a^{2}}-\frac{1}{b^{2}}\right)
$$

The transverse (dipole) wake is then given by $W_{y}(z)=$ $-\int_{-\infty}^{z} d z^{\prime} W_{z}^{(1)}\left(z^{\prime}\right)$. The results of the calculation are shown in Fig. 5. We see that the transverse wake of the long-bottomed transition is also relatively simple, and that the agreement between the nominal and scaled wakes is quite good.

We have shown good agreement between the nominal and scaled wakes for 2D transitions with a short central region and a long one. For the next example we consider a 2D transition with a medium-length central region. The parameters are $a=7.5 \mathrm{~mm}, b=48 \mathrm{~mm}, \theta=5.8^{\circ}$, and length of central region $L=72 \mathrm{~mm}$. We take as nominal bunch length for the calculation $\sigma_{z}=0.5 \mathrm{~mm}$, and again take $\lambda=\frac{1}{2}$ for the scaled calculation. The numerical results are shown in Fig. 6. Compared to the previous example we see nearly the same wake over the bunch, though many

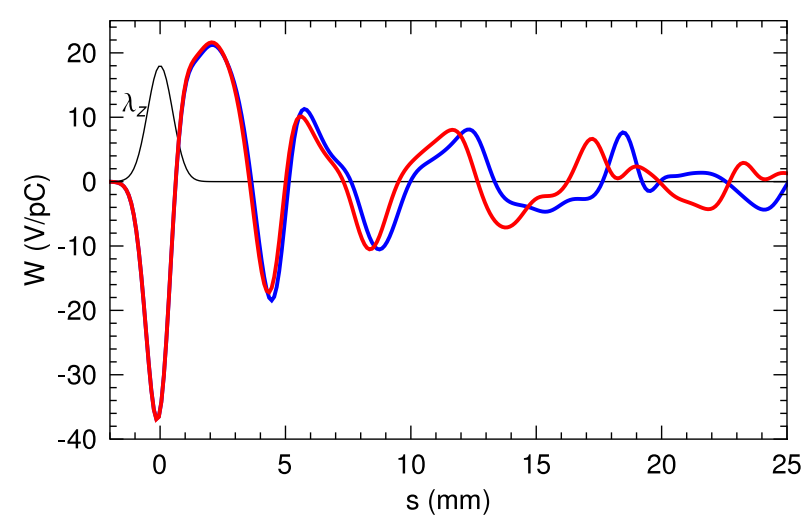

FIG. 6. For the 2D transition with a medium-length central region, and $\sigma_{z}=0.5 \mathrm{~mm}$, the nominal longitudinal wake (blue) and results obtained from the scaled problem with $\lambda=\frac{1}{2}$ (red). The nominal bunch shape is also given with the head to the left (black). more oscillations behind it. Comparing nominal and scaled wakes we again see good agreement near the driving bunch. Farther behind, however, we see that the two wakes gradually deviate from one another, an indication of the accuracy of the scaling.

\section{3D transition}

As a 3D (noncylindrically symmetric) example we consider a longitudinally symmetric, small angle transition, from a large beam pipe to a small one and then back again, with the central region taken to be infinitely long. In the horizontal $(x)$ direction the beam pipe remains unchanged; the transition occurs only in the vertical $(y)$ direction. For the nominal geometry, the large beam pipe has a square cross section of $30 \mathrm{~mm}$ by $30 \mathrm{~mm}(x \times y)$, the small one is rectangular with dimensions $30 \mathrm{~mm}$ by $15 \mathrm{~mm}$, and the central region is assumed to be long. The connecting pipes are straight line tapers (in $y$ ) of angle $3^{\circ}$ (see Fig. 7). The nominal bunch length $\sigma_{z}=0.5 \mathrm{~mm}$. For the scaled case we take $\lambda=\frac{1}{2}$.

The numerical calculations were performed with the program ECHO3D [11]. Like in the 2D long taper example, the total wake of the transition is taken to be the sum of a step-in and a step-out part. (More details of the algorithm ECHO3D uses for such problems can be found in Ref. [13].) The mesh size was taken to be $\sigma_{z} / 10$ in the longitudinal direction and $\sigma_{z} / 5$ in the transverse plane. In Fig. 8 we give the numerical results for the longitudinal wake, and see good agreement between the nominal and scaled results.

For a noncylindrically symmetric structure, but one that has mirror symmetry in $x$ and $y$ (as in our example problem), the transverse (vertical) wake of a beam is given by

$$
W_{y}\left(s, \sigma_{z}\right)=y_{d} W_{y, d}\left(s, \sigma_{z}\right)+y W_{y, q}\left(s, \sigma_{z}\right),
$$

where $W_{y, d}$ is called the dipole wake component and $W_{y, q}$ the quadrupole wake component. Here $y_{d}$ and $y$ are, respectively, the vertical offset - with respect to the axis - of the beam and of a test particle (the offsets of beam and test particle in both $x$ and $y$ are assumed to be small).

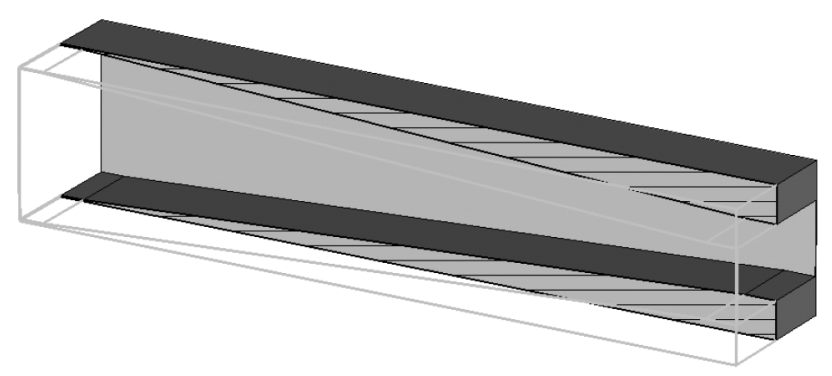

FIG. 7. The 3D test example is a symmetric, small angle transition. Here we show, in cut view, the geometry of one of the tapers of this transition. 


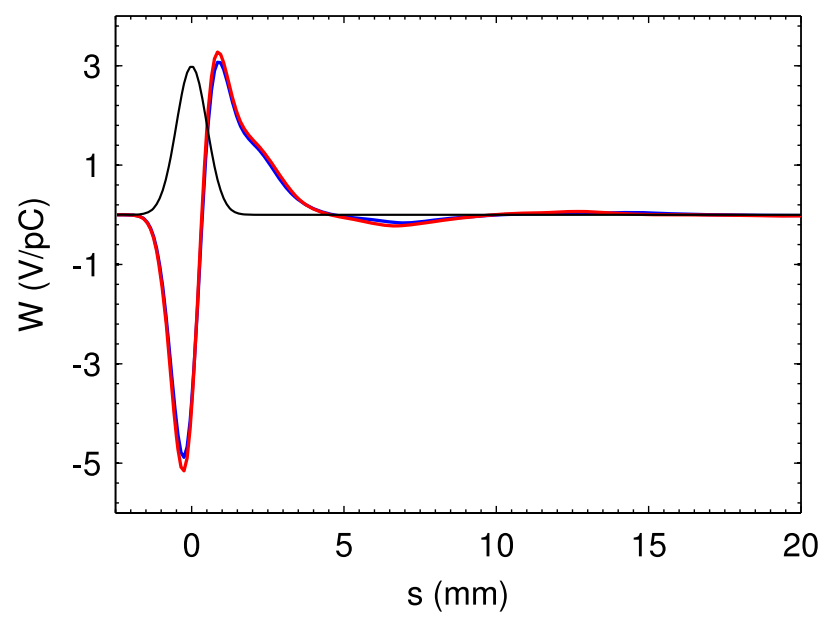

FIG. 8. Longitudinal wake for $\sigma_{z}=0.5 \mathrm{~mm}$ bunch in the 3D collimator (blue) and results obtained from the scaled problem (red).

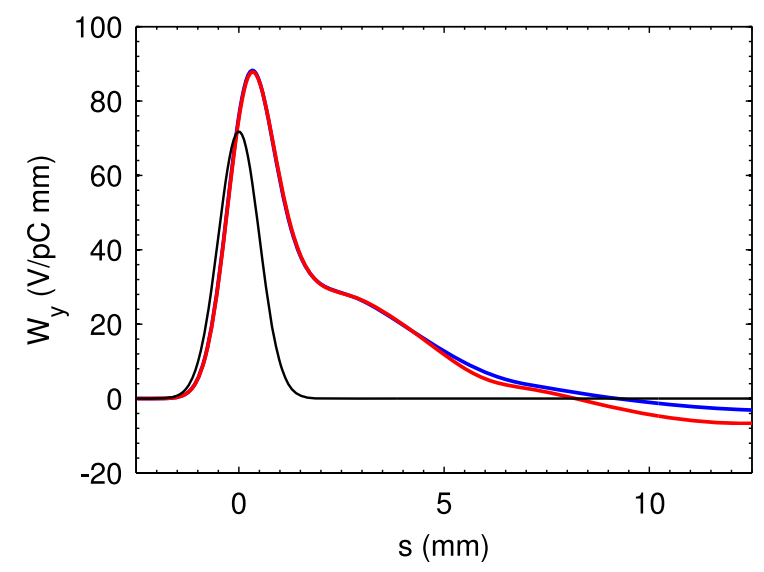

FIG. 9. Dipole component of the transverse wake for $\sigma_{z}=0.5 \mathrm{~mm}$ bunch in the $3 \mathrm{D}$ collimator (blue) and results obtained from the scaled problem (red).

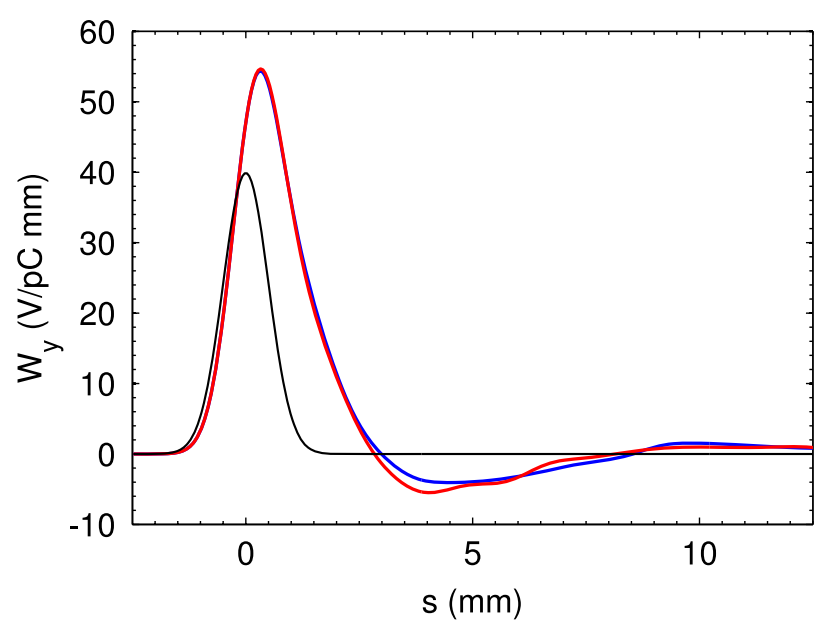

FIG. 10. Quadrupole component of the transverse wake for $\sigma_{z}=0.5 \mathrm{~mm}$ bunch in the $3 \mathrm{D}$ collimator (blue) and results obtained from the scaled problem (red).
The two components of the transverse wake are given in Figs. 9 and 10. We see that in both cases the nominal and scaled wakes agree well.

\section{Discussion}

We need to emphasize that the scaling properties are approximate and valid only within the conditions of applicability. Although we have demonstrated with several examples that they tend to work well, we cannot, for any given geometry, predict in advance their accuracy. For example, in Ref. [14] it was shown that sharp angles in the structure lead to a deterioration in accuracy of Yokoya's model; we also expect that sharp angles will result in less accuracy in our scaling equations, although it is difficult to make a quantitative prediction of the effect. In practice, one can first test the scaling properties with preliminary runs using a relatively crude mesh; if this works well, one can have confidence in using a scaled calculation for the final (and more time consuming) calculations.

\section{CONCLUSIONS}

Based on the parabolic equation approach to Maxwell's equations, we have derived scaling properties of the highfrequency impedance/short bunch wakefields of structures; for the special case of small angle transitions we have shown the scaling properties are valid for all frequencies. Using these scaling properties, one can greatly reduce the calculation time of the wakefield/impedance of long, small angle, beam pipe transitions, like one often finds e.g. in insertion regions of storage rings. We have tested the scaling with wakefield simulations of 2D and 3D models of small angle transitions, and find good agreement from the front of the driving bunch to a significant distance behind it. For wakes that extend further back-such as that of a medium-length $2 \mathrm{D}$ transition example-we see a gradual discrepancy developing far behind the bunch.

\section{ACKNOWLEDGMENTS}

This work was supported by Department of Energy Contract No. DE-AC02-76SF00515 and by the EU Contract No. 011935 EUROFEL.

[1] G. Stupakov, New J. Phys. 8, 280 (2006).

[2] G. Stupakov, Report No. SLAC-PUB-13661, 2009.

[3] G. Stupakov and I. A. Kotelnikov, Phys. Rev. ST Accel. Beams 12, 104401 (2009).

[4] K. Yokoya, Report No. CERN-SL-90-88-AP, 1988.

[5] G. Stupakov, Part. Accel. 56, 83 (1996).

[6] J. Lawson, Report No. RHEL/M 144, 1968.

[7] J. Lawson and M. Sands, Report No. SLAC-PUB-4441, 1987.

[8] G. Stupakov, K. Bane, and I. Zagorodnov, Phys. Rev. ST Accel. Beams 10, 054401 (2007). 
[9] K. Bane, G. Stupakov, and I. Zagorodnov, Phys. Rev. ST Accel. Beams 10, 074401 (2007).

[10] S. A. Heifets and S. Kheifets, Rev. Mod. Phys. 63, 631 (1991).

[11] I. Zagorodnov and T. Weiland, Phys. Rev. ST Accel. Beams 8, 042001 (2005).
[12] Y. Cai et al., Report No. SLAC-PUB-13999, 2010.

[13] I. Zagorodnov, Phys. Rev. ST Accel. Beams 9, 102002 (2006).

[14] B. Podobedov and S. Krinsky, Phys. Rev. ST Accel. Beams 9, 054401 (2006). 